\title{
A Balanced Robertsonian Translocation (13;14) of a Male: A Case Report
}

\author{
Filiz Ozen ${ }^{\mathrm{a}, \mathrm{e}}$, Zuhal Aydan Saglam ${ }^{\mathrm{b}}$, Hande Kucuk Kurtulgan ${ }^{\mathrm{c}}$, Abdul Vahap Ozen ${ }^{\mathrm{d}}$
}

\begin{abstract}
Cytogenetic investigations are of descriptive importance at spontaneous abortus for most couples. The conventional cytogenetic analysis of a couple was performed, who were referred to our Department of Medical Genetics for two recurrent abortions and an intrauterine mort fetale at reproductive history. The clinical characteristics of a woman with normal karyotype $(46, \mathrm{XX})$ and a man with $45, \mathrm{XY}, \mathrm{rob}(13 ; 14)$ are presented under the light of recent literature.
\end{abstract}

Keywords: Habitual abortion; Robertsonian translocation

\section{Introduction}

Recurrent pregnancy loss (RPL) or habitual abortion is defined as spontaneous pregnancy loss at at least two and more pregnancies prior to 20 weeks from the last menstruation [1]. Being an inconsistently defined situation, it is the most common complication of pregnancy [2]. Although 38\% of RPLs are idiopathic, namely they have an unknown etiology, among well-known etiologies, chromosomal abnormalities hold for $50 \%[1,3,4]$.

Cytogenetic studies report that some of chromosomal abnormalities in RPLs result from formation of irregular gametes at parents carrying regular translocation $[5,6]$. The exchange of chromosomal material between two non-homologous chro-

Manuscript accepted for publication August 18, 2014

aDepartment of Medical Genetics, Istanbul Medeniyet University, Goztepe Training and Research Hospital, Turkey

bDepartment of Family Medicine, Istanbul Medeniyet University, Goztepe Training and Research Hospital, Turkey

'Department of Medical Genetics, Sivas Cumhuriyet University Medical Faculty, Turkey

${ }^{\mathrm{d} D e p a r t m e n t}$ of Gynecology and Obstetrics, Istanbul Fatih Sultan Mehmet Training and Research Hospital, Turkey

e Corresponding Author: Filiz Ozen, Department of Medical Genetics, Istanbul Medeniyet University, Goztepe Training and Research Hospital, Turkey. Email: dr.fozen@hotmail.com

doi: http://dx.doi.org/10.14740/jmc1921w mosomes is called reciprocal translocation while if the long arms of two non-homologous acrocentric chromosomes join, the rearrangement is called Robertsonian (ROB) translocation [7]. ROB translocation is the most common structural chromosomal abnormality which has an incidence of $1-1.23 / 1,000$ live births in general population $[4,8]$. Carriers of these translocations do not present with any disorder themselves, yet cause unbalanced chromosomal formation during parental gamete formation.

ROB translocation is caused by joining together of two acrocentric chromosomes which are 13, 14, 15, 21 and 22, while the most common one is between 13 and 14 at a frequency of $75 \%$. At the process the participating chromosomes break at the centromere, and lose their short arms. The long arms join and form a single chromosome with a single centromere or two. The remaining short arms fuse as well and form a reciprocal product which is lost in time within a few cell divisions [8].

These balanced rearrangements do not alter the amount of genetic material, so the carriers of these rearrangements are healthy, normal at phenotype but unaware of the possible results of conception which are recurrent fetal losses, infertility and births with abnormal phenotype due to abnormal segregation during meiosis [9]. Especially male carriers of ROB translocations can have fertility problems presenting with abnormal sperm morphology and low sperm counts [10]. This study reports an ROB translocation $\operatorname{rob}(13 ; 14)$ at a male carrier and RPLs of the couple.

\section{Case Report}

A couple (a 30-year-old male and a 27-year-old female) was referred to our department for cytogenetic investigation with the history of recurrent miscarriages. They have married for 5 years and have not used any contraceptive method so far. The woman's reproductive history consisted of three pregnancies which did not last for more than 3 months. The first abortion took place at the 10th week of her pregnancy. The second pregnancy which was 11 months later resulted with intrauterine mort fetale and the last abortion was at the 13th week of her pregnancy 2 months ago. The couple did not have any blood relation as marriages among siblings of distant relatives may be expected at some regions in Turkey. The physical examination of the couple revealed normal. The woman was healthy, 


\section{IIII}
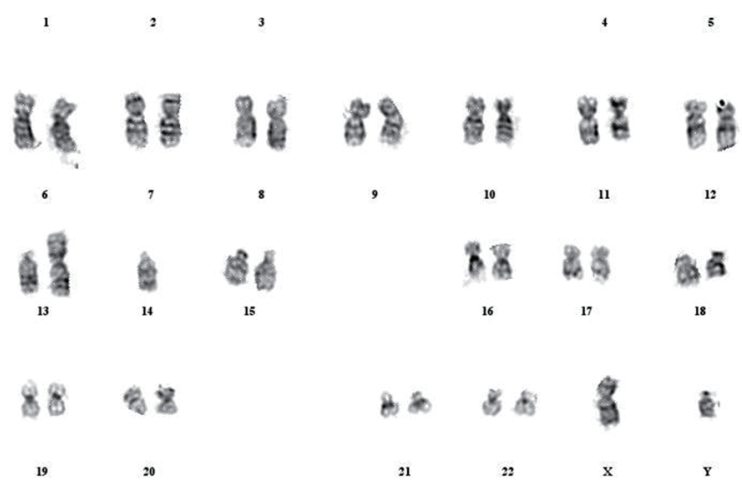

Figure 1. The chromosomal structure of the case, $45, \mathrm{XY}$, rob(13;14).

a non-smoker and had no comorbid diseases. The man was smoking cigarettes (five packs/year). He had chronic B hepatitis infection which was under control at Department of Microbiology and told to be unrelated with his current problem. At the urologic examination, erection and ejaculation were normal. History of repeated abortion was not reported in any other family members of the couple.

\section{Cytogenetic analysis}

The blood sample was collected from the couple in a completely sterile heparinized vacutainer tube and mixed well. The cultures were set up with RPMI 1640 (Rosewell Park Memorial Institute) culture medium. Peripherial blood lymphocytes inducted with $2 \%$ phytohemagglutinin (PHA) were incubated at $37.5{ }^{\circ} \mathrm{C}$ for $72 \mathrm{~h}$. One and a half hours prior to harvest, the cultures were arrested with colchicine and treated with 0.75 $\mathrm{M} \mathrm{KCl}$ (potassium chloride) for $30 \mathrm{~min}$ and fixed in 3:1 ratio of methanol/glacial acetic acid fixative. After air drying, the cultures were set up by Giemsa-trypsin banding (GTB) and chromosomes at 30 - 50 metaphase plates were estimated for the mitotic drive and mitotic index.

Chromosomal analysis of the female partner showed normal 46,XX karyotype. But there was abnormality at the karyotype of the male partner which showed chromosomal constitution of 45,XY, rob(13;14) (Fig. 1).

\section{Discussion}

Mostly being unclinical, approximately $70 \%$ of all conceptions are lost prior to birth [11]. Fifteen percent of pregnancies result with clinically recognized abortus while at $1 \%$, these miscarriages may be silent and habitual as well. Habitual abortus is one of the subjects that discipline of obstetrics fails to define the etiology and prognostic factors [12]. Genetic factors account for $20 \%$ of cases in the etiology which is comprised of parental and fetal chromosomal abnormalities, single gene defects, mutations leading to thrombopathies or any other multifactorial disorders $[4,13]$.

It is believed that in case of at least two recurrent abortions one of the parents carries a balanced chromosomal abnormality $[14,15]$; in general the prevalance is higher in females than males (4i). In the present case, the proband is a male carrier of ROB translocation.

The pregnancies of this couple may have presented as abortus, intrauterine mort fetale, a congenitally anomalous baby, a chromosomally balanced translocation carrier with a normal phenotype or a totally health baby [16] as it is consistent with the reproductive history involving two spontaneous abortions and an intrauterine death and with carriage of ROB translocation which is determined at chromosomal analysis as well.

Cases with recurrent abortions have to be analyzed in order to inform them about genetic possibilites at subsequent pregnancies and offer them a chance for adequate genetic counselling. Preimplantation genetic diagnosis (PGD) is recommended to the carriers of ROB translocation for prenatal diagnosis. This method is expected to decrease the number of inevitable spontaneous abortus $[17,18]$. In our case, the couple is informed about PGD and other prenatal diagnostic approaches which may reduce the frequency of miscarriages for the future pregnancies and is getting genetic counselling.

This case report aims to emphasize the necessity of cytogenetic analysis of couples with recurrent miscarriages in order to exclude the probable presence of any chromosomal rearrangements which are transmitted for many generations without detection in healthy individuals with normal phenotype.

\section{References}

1. Ford HB, Schust DJ. Recurrent pregnancy loss: etiology, diagnosis, and therapy. Rev Obstet Gynecol. 2009;2(2):76-83.

2. Alberman E. The epidemiology of repeated abortion. in: Beard RW, Sharp F. Early pregnancy loss mechanisms and treatment. New York Springer-Verlag, 1988;9-17.

3. Carp HJ. Recurrent miscarriage: genetic factors and assessment of the embryo. Isr Med Assoc J. 2008;10(3):229231.

4. Venkateshwari A, Srilekha A, Sunitha T, Pratibha N, Jyothy A. A Robertsonian Translocation rob $(14 ; 15)$ (q10:q10) in a Patient with Recurrent Abortions: A case report. J Reprod Infertil. 2010;11(3):197-200.

5. Goud TM, Mohammed Al Harassi S, Khalfan Al Salmani K, Mohammed Al Busaidy S, Rajab A. Cytogenetic studies in couples with recurrent miscarriage in the Sultanate of Oman. Reprod Biomed Online. 2009;18(3):424-429.

6. Singh DN, Hara S, Foster HW, Grimes EM. Reproductive performance in women with sex chromosome mosaicism. Obstet Gynecol. 1980;55(5):608-611.

7. Yilmaz A, Zhang XY, Chung JT, Tan SL, Holzer H, Ao 
A. Chromosome segregation analysis in human embryos obtained from couples involving male carriers of reciprocal or Robertsonian translocation. PLoS One. 2012;7(9):e46046.

8. Shuqin X, Dongling T, Kun F, Yanzhi X, Jieping S, Weipeng W, Maowei C, Bo W. Analysis of meiotic segregation patterns and interchromosomal effects in sperm from a Robertsonian translocation family. Biomedical Research. 2014;25(2):233-239.

9. Ogilvie CM, Braude P, Scriven PN. Successful pregnancy outcomes after preimplantation genetic diagnosis (PGD) for carriers of chromosome translocations. Hum Fertil (Camb). 2001;4(3):168-171.

10. Ogur G, Van Assche E, Vegetti W, Verheyen G, Tournaye H, Bonduelle M, Van Steirteghem A, et al. Chromosomal segregation in spermatozoa of 14 Robertsonian translocation carriers. Mol Hum Reprod. 2006;12(3):209-215.

11. Macklon NS, Geraedts JP, Fauser BC. Conception to ongoing pregnancy: the 'black box' of early pregnancy loss. Hum Reprod Update. 2002;8(4):333-343.

12. Clark DA, Lea RG, Podor T, Daya S, Banwatt D, Harley C. Cytokines determining the success or failure of preg- nancy. Ann N Y Acad Sci. 1991;626:524-536.

13. Suzumori N, Sugiura-Ogasawara M. Genetic factors as a cause of miscarriage. Curr Med Chem. 2010;17(29):34313437.

14. Celep F, Karaguzel A, Ozeren M, Bozkaya H. The frequency of chromosomal abnormalities in patients with reproductive failure. Eur J Obstet Gynecol Reprod Biol. 2006;127(1):106-109.

15. Rai R, Regan L. Recurrent miscarriage. Lancet. 2006;368(9535):601-611.

16. Dewald GW, Michels VV. Recurrent miscarriages: cytogenetic causes and genetic counseling of affected families. Clin Obstet Gynecol. 1986;29(4):865-885.

17. Wiland E, Hobel CJ, Hill D, Kurpisz M. Successful pregnancy after preimplantation genetic diagnosis for carrier of $\mathrm{t}(2 ; 7)(\mathrm{p} 11.2 ; \mathrm{q} 22)$ with high rates of unbalanced sperm and embryos: a case report. Prenat Diagn. 2008;28(1):3641.

18. Stephenson MD, Sierra S. Reproductive outcomes in recurrent pregnancy loss associated with a parental carrier of a structural chromosome rearrangement. Hum Reprod. 2006;21(4):1076-1082. 